\title{
Retrospective Review on the Safety and Efficacy of Direct Oral Anticoagulants Compared With Warfarin in Patients With Cirrhosis
}

\author{
Kaitlyn Jones, PharmD; Caroline Pham, PharmD, BCPS; Christine Aguilar, PharmD, BCPS; \\ and Shaila Sheth, PharmD, BCPS
}

\begin{abstract}
Purpose: Patients with cirrhosis needing anticoagulation therapy have historically been prescribed warfarin. New retrospective research has concluded that in patients with cirrhosis direct oral anticoagulants (DOACs) have similar or lower bleeding rates compared with that of warfarin. This study compares the safety and efficacy of DOACs with that of warfarin in patients with cirrhosis.
\end{abstract}

Methods: A retrospective chart review was conducted in adult patients with cirrhosis taking either apixaban, dabigatran, edoxaban, rivaroxaban, or warfarin. Exclusion criteria consisted of patients prescribed triple antithrombotic therapy (dual antiplatelet therapy plus an anticoagulant) and indications other than nonvalvular atrial fibrillation (NVAF) and venous thromboembolism (VTE). The primary endpoint was all-cause bleeding, and the secondary endpoints were failed efficacy and major bleeding as defined by the International Society on Thrombosis and Haemostasis in 2005. Failed efficacy was a combination endpoint including the development of VTE, stroke, myocardial infarction and/or death. Patient data were collected from the Computerized Patient Record System from October 31, 2014 to October 31, 2018.
Results: The study included 42 patients in the DOAC group and 37 patients in the warfarin group. Baseline characteristics were not significantly different between groups except for the Child-Turcotte-Pugh score, Model for End-Stage Liver Disease score, international normalized ratio, and number of days on anticoagulation therapy. The rate of all-cause bleeding in the DOAC group was $16.7 \%(n=7)$ vs $21.6 \%$ $(\mathrm{n}=8)$ in the warfarin group $(P=.7)$. The rate of major bleeding in the DOAC group was $2.4 \%(n=1)$ vs $5.4 \%$ $(n=2)$ in the warfarin group $(P=.6)$. The rate of failed efficacy in the DOAC group was $7.1 \%(n=3)$ compared with $8.1 \%$ $(n=3)$ in the warfarin group $(P=.9)$. Subgroup analysis of allcause bleeding did not identify any significant trends between groups.

Conclusions: There were no statistically significant differences identified between the rates of all-cause bleeding, major bleeding, and failed efficacy between the DOACs and warfarin groups. DOACs may be a safe alternative to warfarin in patients with cirrhosis requiring anticoagulation for NVAF or VTE, but large randomized trials are required to confirm these results.
C oagulation in patients with cirrhosis is a complicated area of evolving research. Patients with cirrhosis were originally thought to be naturally anticoagulated due to the decreased production of clotting factors and platelets, combined with an increased international normalized ratio (INR). ${ }^{1}$ New data have shown that patients with cirrhosis are at a concomitant risk of bleeding and thrombosis due to increased platelet aggregation, decreased fibrinolysis, and decreased production of natural anticoagulants such as protein $C$ and antithrombin. ${ }^{1}$ Traditionally, patients with cirrhosis needing anticoagulation therapy for comorbid conditions, such as nonvalvular atrial fibrillation (NVAF) or venous thromboembolism (VTE) were placed on warfarin therapy. Managing warfarin in patients with cirrhosis poses a challenge to clinicians due to the many food and drug interactions, narrow therapeutic index, and complications with maintaining a therapeutic INR. ${ }^{1}$
Direct oral anticoagulants (DOACs) have Kaitlyn Jones is a Clinical several benefits over warfarin therapy, includ- Pharmacy Specialist in ing convenience, decreased monitoring, de- Primary Care at the University creased drug and dietary restrictions, and faster onset of action. ${ }^{2}$ Conversely, DOACs undergo extensive hepatic metabolism giving rise to concerns about supratherapeutic drug levels and increased bleeding rates in patients with liver dysfunction. ${ }^{1}$ Consequently, patients with cirrhosis were excluded from the pivotal trials establishing DOACs for NVAF and VTE treatment. Exclusion of these patients in major clinical trials alongside the challenges of managing warfarin warrant an evaluation of the efficacy and safety of DOACs in patients with cirrhosis.

Recent retrospective studies have examined the use of DOACs in patients with doi:10.12788/fp.0058 cirrhosis and found favorable results. A retrospective chart review by Intagliata and colleagues consisting of 39 patients with cirrhosis using either a DOAC or warfarin found similar rates of all-cause bleeding and major bleeding between the 2 groups. ${ }^{3}$ 
TABLE 1 Baseline Characteristics

\begin{tabular}{|c|c|c|c|}
\hline Characteristics & $\begin{array}{c}\text { DOAC } \\
(n=42)\end{array}$ & $\begin{array}{c}\text { Warfarin } \\
(\mathrm{n}=37)\end{array}$ & $P$ Value \\
\hline Male sex, No. (\%) & $41(97.6)$ & $37(100)$ & .99 \\
\hline Age, mean (SD), y & $71.9(6.2)$ & $70.3(7.5)$ & .32 \\
\hline Weight, mean (SD), kg & $93(19.6)$ & $99(23.8)$ & .22 \\
\hline Body mass index, mean (SD) & $28.8(6.1)$ & $31.1(7)$ & .12 \\
\hline \multicolumn{4}{|l|}{ Etiology of cirrhosis, No. (\%) } \\
\hline Alcohol & $9(21.4)$ & $8(21.6)$ & .99 \\
\hline Mixed & $10(23.8)$ & $8(21.6)$ & .99 \\
\hline Nonalchoholic fatty liver disease & $10(23.8)$ & $9(24.3)$ & .99 \\
\hline Viral & $12(28.6)$ & $11(29.7)$ & .99 \\
\hline Other & $1(2.4)$ & $1(2.7)$ & .99 \\
\hline \multicolumn{4}{|l|}{ Child-Turcotte-Pugh score, No. (\%) } \\
\hline A & $34(81)$ & $16(43.2)$ & $.0009^{a}$ \\
\hline $\mathrm{B}$ & $8(19.1)$ & $19(51.4)$ & $.004^{\mathrm{a}}$ \\
\hline $\mathrm{C}$ & 0 & $2(5.4)$ & .23 \\
\hline MELD score, mean (SD) & $9.4(4.3)$ & $16.3(7.1)$ & $.0001^{\mathrm{a}}$ \\
\hline $\mathrm{CHA}_{2} \mathrm{DS}_{2}-\mathrm{VASc}$ score, mean (SD) & $3.6(1.5)$ & $3.7(1.2)$ & .88 \\
\hline Congestive heart failure, No. (\%) & $16(38.1)$ & $17(46)$ & .46 \\
\hline Hypertension, No. (\%) & $27(64.3)$ & $23(62.2)$ & .99 \\
\hline Diabetes mellitus, No. (\%) & $11(26.2)$ & 15 (40.5) & .20 \\
\hline Prior stroke, transient ischemic & & & \\
\hline attack, or thromboembolism, №. (\%) & $7(16.7)$ & $7(18.9)$ & .99 \\
\hline Vascular disease, No. (\%) & $12(28.6)$ & 7 (18.9) & .41 \\
\hline Age $65-74$, No. (\%), y & $19(45.2)$ & $15(40.5)$ & .80 \\
\hline Age $\geq 75$, No. (\%), y & $10(23.8)$ & $8(21.6)$ & .99 \\
\hline \multicolumn{4}{|l|}{ Indications for anticoagulation, No. (\%) } \\
\hline Atrial fibrillation & 33 (78.6) & $29(78.4)$ & .99 \\
\hline Venous thromboembolism & $9(21.4)$ & $8(21.6)$ & .99 \\
\hline Deep vein thrombosis & $4(9.5)$ & 7 (18.9) & --- \\
\hline Portal vein thrombosis & 0 & $1(2.7)$ & --- \\
\hline Pulmonary embolism & $5(11.9)$ & $1(2.7)$ & --- \\
\hline \multicolumn{4}{|l|}{ Medications, No. (\%) } \\
\hline Aspirin & $21(50)$ & $20(54.1)$ & .82 \\
\hline Thienopyridine & $1(2.4)$ & $3(8.1)$ & .34 \\
\hline Nonsteroidal anti-inflammatory drug & $2(4.8)$ & $2(5.4)$ & .99 \\
\hline Proton pump inhibitor & 22 (52.4) & $16(43.2)$ & .50 \\
\hline Histamine-2 receptor antagonist & $4(9.5)$ & $3(8.1)$ & .99 \\
\hline \multicolumn{4}{|l|}{ Concominant conditions, No. (\%) } \\
\hline Esophageal varices & $8(19.1)$ & $5(13.5)$ & .56 \\
\hline Gastric varices & $1(2.4)$ & $2(5.4)$ & .60 \\
\hline Active malignancy & 5 (11.9) & $1(2.7)$ & .21 \\
\hline \multicolumn{4}{|l|}{ Laboratory values, mean (SD) } \\
\hline Albumin, $\mathrm{g} / \mathrm{dL}$ & $3.7(0.4)$ & $3.5(0.5)$ & .29 \\
\hline Total bilirubin, $\mathrm{mg} / \mathrm{dL}$ & $1(0.5)$ & $1.2(0.9)$ & .19 \\
\hline Creatinine, $\mathrm{mg} / \mathrm{dL}$ & $1.1(0.4)$ & $1.6(1.6)$ & .10 \\
\hline International normalized ratio & $1.3(0.4)$ & $2.1(0.9)$ & $.0001^{a}$ \\
\hline Platelets, $\mathrm{k} / \mathrm{dL}$ & $170.6(53)$ & $170.6(118.9)$ & .99 \\
\hline \multicolumn{4}{|l|}{ Anticoagulant medications, №. (\%) } \\
\hline Apixaban & $29(69.1)$ & --- & --- \\
\hline Dabigatran & $4(9.5)$ & --- & --- \\
\hline Edoxaban & 0 & --- & --- \\
\hline Rivaroxaban & $9(21.4)$ & --- & --- \\
\hline Anticoagulation therapy, mean (SD), d & $500.4(432.3)$ & $1,652.4(1,642.1)$ & $.0001^{a}$ \\
\hline
\end{tabular}

Abbreviations: DOAC, direct oral anticoagulant; MELD, Model for End-Stage Liver Disease. aStatistically significant.
A retrospective cohort study by Hum and colleagues consisting of 45 patients with cirrhosis compared the use of DOACs with warfarin or low-molecular weight heparin (LMWH). ${ }^{4}$ Hum and colleagues found patients prescribed a DOAC had significantly fewer major bleeding events than did patients using warfarin or $\mathrm{LMWH} .{ }^{4}$ The largest retrospective cohort study consisted of $233 \mathrm{pa}$ tients with chronic liver disease and found no differences among all-cause bleeding and major bleeding rates between patients using DOACs compared with those of patients using warfarin. ${ }^{5}$

The purpose of this research is to evaluate the safety and efficacy of DOACs in veteran patients with cirrhosis compared with patients using warfarin.

\section{METHODS}

A retrospective single-center chart review was conducted at the Michael E. DeBakey Veterans Affairs Medical Center (MEDVAMC) in Houston, Texas, between October 31, 2014 and October 31, 2018. Patients included in the study were adults aged $\geq 18$ years with a diagnosis of cirrhosis and prescribed any of the following oral anticoagulants: apixaban, dabigatran, edoxaban, rivaroxaban, or warfarin. Patients prescribed apixaban, dabigatran, edoxaban, or rivaroxaban were collectively grouped into the DOAC group, while patients prescribed warfarin were classified as the standard of care comparator group.

A diagnosis of cirrhosis was confirmed using a combination of the codes from the ninth and tenth editions of the International Classification of Diseases (ICD) for cirrhosis, documentation of diagnostic confirmation by clinicians from the gastroenterology or hepatology services, and positive liver biopsy result. Liver function tests, liver ultrasound results, and FibroSure biomarker assays were used to aid in confirming the diagnosis of cirrhosis but were not considered definitive. Patients were excluded from the trial if they had indications for anticoagulation other than NVAF and VTE and/or were prescribed triple antithrombotic therapy (dual antiplatelet therapy plus an anticoagulant). Patients who switched anticoagulant therapy during the trial period (ie, switched from warfarin to a DOAC) were also excluded from the analysis. 
Patient demographic characteristics that were collected included weight; body mass index (BMI); etiology of cirrhosis; ChildTurcotte-Pugh, Model for End-Stage Liver Disease (MELD), and CHA $\mathrm{DS}_{2}$-VASc score; concomitant antiplatelet, nonsteroidal antiinflammatory drug (NSAID), proton pump inhibitor (PPI), and histamine-2 receptor antagonist (H2RA) medications; presence of gastric and/or esophageal varices; active malignancies; albumin, total bilirubin, serum creatinine, INR, and platelet laboratory values; and indication and duration of anticoagulation therapy.

Two patient lists were used to identify patients for inclusion in the warfarin arm. The first patient list was generated using the US Department of Veterans Affairs (VA) Cirrhosis Tracker, which identified patients with an ICD-9/10 code for cirrhosis and an INR laboratory value. Patients generated from the VA Cirrhosis Tracker with an INR $>1.5$ were screened for a warfarin prescription and then evaluated for full study inclusion. The second patient list was generated using the VA Advanced Liver Disease Dashboard which identified patients with ICD-9/10 codes for advanced liver disease and an active warfarin prescription. Patients with an active warfarin prescription were then evaluated for full study inclusion. A single patient list was generated to identify patients for inclusion in the DOAC arm. This patient list was generated using the VA DOAC dashboard, which identified patients with an active DOAC prescription and an ICD-9/10 code for cirrhosis. Patients with an ICD-9/10 code for cirrhosis and prescribed a DOAC were screened for full study inclusion. Patient data were collected from the MEDVAMC Computerized Patient Record System (CPRS) electronic health record (EHR). The research study was approved by the Baylor College of Medicine Institutional Review Board and the VA Office of Research and Development.

\section{Outcomes}

The primary endpoint for the study was allcause bleeding. The secondary endpoints for the study were major bleeding and failed efficacy. Major bleeding was defined using the International Society on Thrombosis and Haemostasis (ISTH) 2005 definition: fatal bleeding, symptomatic bleeding in a criti-
TABLE 2 Primary and Secondary Outcomes

\begin{tabular}{lccc} 
Safety Outcomes, No. (\%) & DOAC $(\mathbf{n}=\mathbf{4 2})$ & Warfarin $(\mathbf{n}=\mathbf{3 7})$ & $\boldsymbol{P}$ Value \\
\hline All-cause bleeding & $7(16.7)$ & $8(21.6)$ & .77 \\
\hline Major bleeding & $1(2.4)$ & $2(5.4)$ & .60 \\
\hline Nonmajor bleeding & $6(14.3)$ & $6(16.2)$ & .99 \\
\hline Efficacy Outcomes, No. (\%) & & & .99 \\
\hline Failed efficacy & $3(7.1)$ & $3(8.1)$ & .50 \\
\hline Thrombosis & $2(4.8)$ & 0 & .99 \\
\hline Stroke & 0 & 0 & .99 \\
\hline Myocardial infarction & $1(2.4)$ & 0 & .10 \\
\hline Death (all-cause) & 0 & $3(8.1)$ & \\
\hline
\end{tabular}

Abbreviation: DOAC, direct oral anticoagulant.

cal organ area (ie, intracranial, intraspinal, intraocular, retroperitoneal, intraarticular, pericardial, or intramuscular with compartment syndrome), or bleeding causing a fall in hemoglobin level of $>2 \mathrm{~g} / \mathrm{dL}$ or leading to the transfusion of $\geq 2$ units of red cells. ${ }^{6}$ Failed efficacy was a combination endpoint that included development of VTE, stroke, myocardial infarction (MI), and/or death. A prespecified subgroup analysis was conducted at the end of the study period to analyze trends in the DOAC and warfarin groups with respect to all-cause bleeding. All-cause bleeding risk was stratified by weight, BMI, Child-Turcotte-Pugh score, MELD score, presence of gastric and/or esophageal varices, active malignancies, percentage of time within therapeutic INR range in the warfarin group, indications for anticoagulation, and antiplatelet, NSAID, PPI, and H2RA therapy.

\section{Statistical Analysis}

Data were analyzed using descriptive and inferential statistics. Continuous data were analyzed using the Student $t$ test, and categorical data were analyzed using the Fisher exact test. Previous studies determined an all-cause bleeding rate of 10 to $17 \%$ for warfarin compared with $5 \%$ for DOACs. ${ }^{7,8}$ To detect a $12 \%$ difference in the all-cause bleeding rate between DOACs and warfarin, 212 patients would be needed to achieve $80 \%$ power at an $\alpha$ level of 0.05 . 
FIGURE Study Participants in Comparison of Safety and Efficacy of Anticoagulants in Patients With Cirrhosis

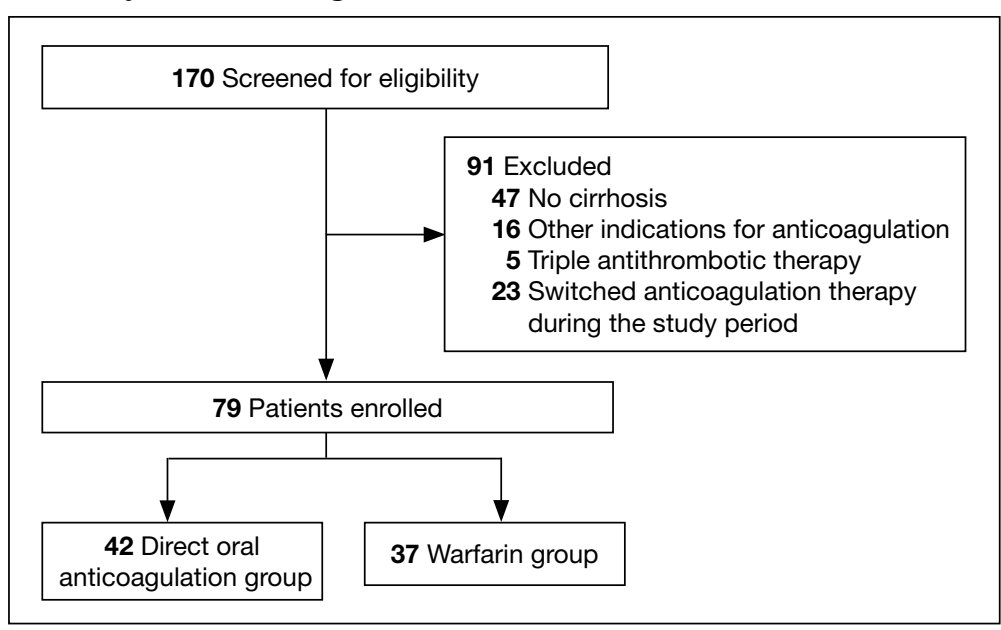

RESULTS

A total of 170 patients were screened, and after applying inclusion and exclusion criteria, 79 patients were enrolled in the study (Figure). The DOAC group included 42 patients, and the warfarin group included 37 patients. In the DOAC group, 69.1\% ( $\mathrm{n}=$ 29) of patients were taking apixaban, $21.4 \%$ $(\mathrm{n}=9)$ rivaroxaban, and $9.5 \%(\mathrm{n}=4)$ dabigatran. There were no patients prescribed edoxaban during the study period

Baseline characteristics were similar between the 2 groups except for ChildTurcotte-Pugh score, MELD score, mean INR, and number of days on anticoagulation therapy (Table 1). Most of the patients were male $(98.7 \%)$, and the mean age was 71 years. The most common causes of cirrhosis were viral (29.1\%), nonalcoholic fatty liver disease (NAFLD) (24.1\%), multiple causes $(22.8 \%)$, and alcohol (21.5\%). Sixtytwo patients (78.5\%) had a NVAF indication for anticoagulation. The average $\mathrm{CHA}_{2} \mathrm{DS}_{2}$ VASc score was 3.7. Aspirin was prescribed in $51.9 \%(n=41)$ of patients, and PPIs were prescribed in $48.1 \%(\mathrm{n}=38)$ of patients. At inclusion, esophageal varices were present in 13 patients and active malignancies were present in 6 patients.

Statistically significant differences in baseline characteristics were found between mean INR, Child-Turcotte-Pugh scores, MELD scores, and number of days on anticoagulant therapy. The mean INR was 1.3 in the DOAC group compared with 2.1 in the war- farin group $(P=.0001)$. Eighty-one percent $(\mathrm{n}=34)$ of patients in the DOAC group had a Child-Turcotte-Pugh score of A compared with $43.2 \%(\mathrm{n}=16)$ of patients in the warfarin group $(P=.0009)$. Eight patients in the DOAC group had a Child-Turcotte-Pugh score of B compared with 19 patients in the warfarin group $(P=.004)$. The mean MELD score was 9.4 in the DOAC group compared with 16.3 in the warfarin group $(P=.0001)$. The mean days on anticoagulant therapy was 500.4 days for the DOAC group compared with 1,652.4 days for the warfarin group $(P=.0001)$.

\section{Safety Outcome}

The primary outcome comparing all-cause bleeding rates between patients on DOACs compared with warfarin are listed in Table 2 . With respect to the primary outcome, $7(16.7 \%)$ patients on DOACs experienced a bleeding event compared with 8 (21.6\%) patients on warfarin $(P=.77)$. No statistically significant differences were detected between the DOAC and warfarin groups with respect to all-cause bleeding. Seven bleeding events occurred in the DOAC group; 1 met the qualification for major bleeding with a suspected gastrointestinal (GI) bleed. ${ }^{6}$ The other 6 bleeding episodes in the DOAC group consisted of hematoma, epistaxis, hematuria, and hematochezia. Eight bleeding events occurred in the warfarin group; 2 met the qualification for major bleeding with an intracranial hemorrhage and upper GI bleed. ${ }^{6}$ The other 6 bleeding episodes in the warfarin group consisted of epistaxis, bleeding gums, hematuria, and hematochezia. There were no statistically significant differences between the rates of major bleeding and nonmajor bleeding between the DOAC and warfarin groups.

\section{Efficacy Outcomes}

There were 3 events in the DOAC group and 3 events in the warfarin group $(P=.99)$. In the DOAC group, 2 patients experienced a pulmonary embolism, and 1 patient experienced a MI. In the warfarin group, 3 patients died (end-stage heart failure, unknown cause due to death at an outside hospital, and sepsis/organ failure). There were no statistically significant differences between the composite endpoint of failed efficacy or the 
TABLE 3 Subgroup Analysis All-Cause Bleeding

\begin{tabular}{|c|c|c|c|c|}
\hline \multirow[b]{2}{*}{ Characteristics } & \multicolumn{2}{|c|}{ DOAC } & \multicolumn{2}{|c|}{ Warfarin } \\
\hline & $\begin{array}{l}\text { All-Cause Bleeding } \\
\qquad(n=7)\end{array}$ & $\begin{array}{l}\text { No Bleeding } \\
(n=35)\end{array}$ & $\begin{array}{l}\text { All-Cause Bleeding } \\
\qquad(\mathrm{n}=8)\end{array}$ & $\begin{array}{l}\text { No Bleeding } \\
(n=29)\end{array}$ \\
\hline Weight, mean (SD), kg & $94.4(11.3)$ & $92.7(21)$ & $100.8(27.2)$ & $98.5(23.3)$ \\
\hline Body mass index, mean (SD) & $29.2(2.7)$ & $28.7(6.6)$ & $31.7(7.1)$ & $30.9(7.1)$ \\
\hline \multicolumn{5}{|l|}{ Child-Turcotte-Pugh score, No. (\%) } \\
\hline$A$ & $5(71.4)$ & $29(82.9)$ & $3(37.5)$ & $13(44.8)$ \\
\hline B & $2(28.6)$ & $6(17.1)$ & $4(50)$ & $15(51.7)$ \\
\hline C & 0 & 0 & $1(12.5)$ & $1(3.5)$ \\
\hline MELD score, mean (SD) & $9.9(2.3)$ & $9.3(4.6)$ & $20.6(8.1)$ & $15.2(6.5)$ \\
\hline \multicolumn{5}{|l|}{ Anticoagulation indication, No. (\%) } \\
\hline Atrial fibrillation & $5(71.4)$ & $28(80)$ & $8(100)$ & $21(72.4)$ \\
\hline Venous thromboembolism & $2(28.6)$ & $7(20)$ & 0 & $8(27.6)$ \\
\hline \multicolumn{5}{|l|}{ Medications, №. (\%) } \\
\hline Aspirin & $3(42.9)$ & $18(51.4)$ & $4(50)$ & $16(55.2)$ \\
\hline Thienopyridine & 0 & $1(2.9)$ & $1(12.5)$ & $2(6.9)$ \\
\hline Nonsteroidal anti-inflammatory drug & 0 & $2(5.7)$ & 0 & $2(6.9)$ \\
\hline Proton pump inhibitors & $2(28.6)$ & $20(57.1)$ & $5(62.5)$ & $11(37.9)$ \\
\hline Histamine-2 receptor antagonist & 0 & $4(11.4)$ & $2(25)$ & $1(3.5)$ \\
\hline \multicolumn{5}{|l|}{ Concominant conditions, No. (\%) } \\
\hline Esophageal varices & $1(14.3)$ & $7(20)$ & $1(12.5)$ & $4(13.8)$ \\
\hline Gastric varices & 0 & $1(2.9)$ & $2(25)$ & 0 \\
\hline Active malignancy & 0 & $4(11.4)$ & 0 & $1(3.5)$ \\
\hline \multicolumn{5}{|l|}{ Anticoagulant medications, №. (\%) } \\
\hline Apixaban & $3(42.9)$ & $26(74.3)$ & -- & -- \\
\hline Dabigatran & $2(28.6)$ & $2(5.7)$ & -- & -- \\
\hline Rivaroxaban & $2(28.6)$ & $7(20)$ & -- & -- \\
\hline Time within therapeutic INR range, mean (SD), \% & -- & -- & $38.8(31.8)$ & 60 (22) \\
\hline
\end{tabular}

Abbreviations: DOAC, direct oral anticoagulant; INR, international normalized ratio; MELD, Model for End-Stage Liver Disease; NAFLD, nonalchoholic fatty liver disease.

individual endpoints of VTE, stroke, MI, and death.

\section{Subgroup Analysis}

A prespecified subgroup analysis was conducted to determine risk factors for all-cause bleeding within each treatment group (Table 3 ). No significant trends were observed in the following risk factors: Child-TurcottePugh score, indication for anticoagulation, use of NSAIDs, PPIs or H2RAs, presence of gastric or esophageal varices, active malignancies, and time within therapeutic INR range in the warfarin group. Patients with bleeding events had slightly increased weight and BMI vs patients without bleeding events. Within the warfarin group, patients with bleeding events had slightly elevated MELD scores compared to patients without bleeding events. There was an equal balance of patients prescribed aspirin therapy between the groups with and without bleeding events. Overall, no significant risk factors were identified for all-cause bleeding.

\section{DISCUSSION}

Initially, patients with cirrhosis were excluded from DOAC trials due to concerns for increased bleeding risk with hepatically eliminated medications. New retrospective research has concluded that in patients with cirrhosis, DOACs have similar or lower bleeding rates when compared directly to warfarin.., 10 
In this study, no statistically significant differences were detected between the primary and secondary outcomes of all-cause bleeding, major bleeding, or failed efficacy. Subgroup analysis did not identify any significant risk factors with respect to all-cause bleeding among patients in the DOAC and warfarin groups. To meet $80 \%$ power, 212 patients needed to be enrolled in the study; however, only 79 patients were enrolled, and power was not met. The results of this study should be interpreted cautiously as hypothesisgenerating due to the small sample size. Strengths of this study include similar baseline characteristics between the DOAC and warfarin groups, 4-year length of retrospective data review, and availability of both inpatient and outpatient EHR limiting the amount of missing data points.

Baseline characteristics were similar between the groups except for mean INR, Child-Turcotte-Pugh score, MELD score, and number of days on anticoagulation therapy. The difference in mean INR between groups is expected as patients in the warfarin group have a goal INR of 2 to 3 to maintain therapeutic efficacy and safety. INR is not used as a marker of efficacy or safety with DOACs; therefore, a consistent elevation in INR is not expected. ChildTurcotte-Pugh scores are calculated using INR levels. ${ }^{11}$ When calculating the score, patients with an INR $<1.7$ receive 1 point; patients with an INR between 1.7 and 2.3 receive 2 points. ${ }^{11}$ Therefore, patients in the warfarin group will have artificially inflated Child-Turcotte-Pugh scores as this group has goal INR levels of 2 to 3 . This makes Child-Turcotte-Pugh scores unreliable markers of disease severity in patients using warfarin therapy. When the INR scores for patients prescribed warfarin were replaced with values $<1.7$, the statistical difference disappeared between the warfarin and DOAC groups. The same effect is seen on MELD scores for patients prescribed warfarin therapy. The MELD score is calculated using INR levels. ${ }^{12}$ MELD scores also will be artificially elevated in patients prescribed warfarin therapy due to the INR elevation to between 2 and 3. When MELD scores for patients prescribed warfarin were replaced with values similar to those in the DOAC group, the statistical difference disappeared between the warfarin and DOAC groups.

The last statistically significant difference was found in number of days on anticoagulant therapy. This difference was expected as warfarin is the standard of care for anticoagulation treatment in patients with cirrhosis. The first DOAC, dabigatran, was not approved by the US Food and Drug Administration until 2010. ${ }^{13}$ DOACs have only recently been used in patients with cirrhosis accounting for the statistically significant difference in days on anticoagulation therapy between the warfarin and DOAC groups.

\section{Limitations}

The inability to meet power or evaluate adherence and appropriate renal dose adjustments for DOACs limited this study. This study was conducted at a single center in a predominantly male veteran population and therefore may not be generalizable to other populations. A majority of patients in the DOAC group were prescribed apixaban $(69.1 \%)$, which may have affected the overall rate of major bleeding in the DOAC group. Pivotal trials of apixaban have shown a consistent decreased risk of major bleeding in patients with NVAF or VTE when compared with warfarin. ${ }^{14,15}$ Therefore, the results of this study may not be generalizable to all DOACs.

An inherent limitation of this study was the inability to collect data verifying adherence in the DOAC group. However, in the warfarin group, percentage of time within the therapeutic INR range of 2 to 3 was collected. While not a direct marker of adherence, this does allow for limited evaluation of therapeutic efficacy and safety within the warfarin group. Last, proper dosing of DOACs in patients with and without adequate renal function was not evaluated in this study.

\section{CONCLUSIONS}

The results of this study are consistent with other retrospective research and literature reviews. There were no statistically significant differences identified between the rates of all-cause bleeding, major bleeding, and failed efficacy between the DOAC and warfarin groups. DOACs may be a safe alternative to warfarin in patients with cirrhosis 
requiring anticoagulation for NVAF or VTE, but large randomized trials are required to confirm these results.

\section{Author disclosures}

The authors report no actual or potential conflicts of interest with regard to this article.

\section{Disclaimer}

The opinions expressed herein are those of the authors and do not necessarily reflect those of Federal Practitioner, Frontline Medical Communications Inc., the US Government, or any of its agencies. This article may discuss unlabeled or investigational use of certain drugs. Please review the complete prescribing information for specific drugs or drug combinations - including indications, contraindications, warnings, and adverse effects-before administering pharmacologic therapy to patients.

\section{References}

1. Qamar A, Vaduganathan M, Greenberger NJ, Giugliano RP. Oral anticoagulation in patients with liver disease. J Am Coll Cardiol. 2018;71(19):2162-2175. doi:10.1016/j.jacc.2018.03.023

2. Priyanka P, Kupec JT, Krafft M, Shah NA, Reynolds GJ. Newer oral anticoagulants in the treatment of acute portal vein thrombosis in patients with and without cirrhosis. Int $J$ Hepatol. 2018;2018:8432781. Published 2018 Jun 5. doi:10.1155/2018/8432781

3. Intagliata NM, Henry $\mathrm{ZH}$, Maitland $\mathrm{H}$, et al. Direct oral anticoagulants in cirrhosis patients pose similar risks of bleeding when compared to traditional anticoagulation. Dig Dis Sci. 2016;61(6):1721-1727. doi:10.1007/s10620-015-4012-2

4. Hum J, Shatzel JJ, Jou JH, Deloughery TG. The efficacy and safety of direct oral anticoagulants vs traditional anticoagulants in cirrhosis. Eur J Haematol. 2017;98(4):393-397. doi:10.1111/ejh.12844

5. Goriacko P, Veltri KT. Safety of direct oral anticoagulants vs warfarin in patients with chronic liver disease and atrial fibrillation. Eur J Haematol. 2018;100(5):488-493. doi:10.1111/ejh.13045

6. Schulman S, Kearon C; Subcommittee on Control of Anticoagulation of the Scientific and Standardization Committee of the International Society on Thrombosis and Haemostasis. Definition of major bleeding in clinical investigations of antihemostatic medicinal products in nonsurgical patients. J Thromb Haemost. 2005;3(4):692-694. doi:10.1111/j.1538-7836.2005.01204.x

7. Rubboli A, Becattini C, Verheugt FW. Incidence, clinical impact and risk of bleeding during oral anticoagulation therapy. World J Cardiol. 2011;3(11):351-358. doi:10.4330/wjc.v3.i11.351

8. Ruff CT, Giugliano RP, Braunwald E, et al. Comparison of the efficacy and safety of new oral anticoagulants with warfarin in patients with atrial fibrillation: a meta-analysis of randomised trials. Lancet. 2014;383(9921):955-962. doi:10.1016/S0140-6736(13)62343-0

9. Hoolwerf EW, Kraaijpoel N, Büller HR, van Es N. Direct oral anticoagulants in patients with liver cirrhosis: $A$ systematic review. Thromb Res. 2018;170:102-108. doi:10.1016/j.thromres.2018.08.011

10. Steuber TD, Howard ML, Nisly SA. Direct oral anticoagulants in chronic liver disease. Ann Pharmacother. 2019;53(10):1042-1049. doi:10.1177/1060028019841582

11. Janevska D, Chaloska-Ivanova V, Janevski V. Hepatocellular carcinoma: risk factors, diagnosis and treatment. Open Access Maced J Med Sci. 2015;3(4):732-736. doi:10.3889/oamims.2015.111

12. Singal AK, Kamath PS. Model for End-Stage Liver Disease. J Clin Exp Hepatol. 2013;3(1):50-60. doi:10.1016/j.jceh.2012.11.002

13. Joppa SA, Salciccioli J, Adamski J, et al. A practical review of the emerging direct anticoagulants, laboratory monitoring, and reversal agents. J Clin Med. 2018;7(2):29. Published 2018 Feb 11. doi:10.3390/jcm7020029

14. Granger CB, Alexander JH, McMurray JJ, et al. Apixaban versus warfarin in patients with atrial fibrillation. N Engl J Med. 2011;365(11):981-992. doi:10.1056/NEJMoa1107039

15. Agnelli G, Buller HR, Cohen A, et al. Oral apixaban for the treatment of acute venous thromboembolism. N Engl J Med. 2013;369(9):799-808. doi:10.1056/NEJMoa1302507 